\title{
Assessment of pharmacokinetics and biological effects of lignin- derived polyphenolic compositions BP-C3 and BP-C2 in in vivo models
}

\author{
Panchenko A.V. ${ }^{1}$, Fedoros E.I. ${ }^{1,2}$, Pigarev S.E. ${ }^{1,2}$, Maydin M.A. ${ }^{1}$, Gubareva E.A. ${ }^{1}$, \\ Bykov V.N. ${ }^{1}$, Drachev I.S. ${ }^{1}$, Anisimov V.N. ${ }^{1}$, Chernysheva M.G. ${ }^{3}$, Badun G.A. ${ }^{3}$, \\ Perminova I.V. ${ }^{3}$ \\ ${ }^{1}$ Petrov National Medical Research Center of Oncology, Saint Petersburg, Russia, \\ ando.pan@gmail.com \\ ${ }^{2}$ Nobel, Ltd., Saint Petersburg, Russia \\ ${ }^{3}$ Lomonosov Moscow State University, Moscow, Russia
}

Keywords: lignin, polyphenolic composition, BP-C3, BP-C2, pharmacokinetics, effects

doi: 10.36291/HIT.2019.panchenko.096

BP-Cx-1 is a water-soluble multicomponent composition of polyphenolic compounds derived from lignin-based enterosorbent - Polyphepan ${ }^{\circledR}$ treated in the alkaline medium at the elevated pressure and temperature with continuous supply of oxygen [1]. BP-Cx-1 is used as base for a number of compositions with different activities. BP-C3 is a formulation, which comprises BP-Cx-1 with iron complex, selenium, ascorbic acid and retinol. Longterm treatment with BP-C3 had anticarcinogenic and geroprotective activity in female SHR mice [2]. BP-C3 was found to reduce the toxic effect of 5-fluorouracil against hematopoiesis and intestinal epithelium which was evident by preserved organ/body ratios for the lymphopoietic organs, anemia reduction and intestinal crypt survival [3]. BP-C2 is a composition which comprises BP-Cx-1 with ammonium molybdate, developed as radioprotector/radiomitigator [4]. BP-C2 was tested in two total body gamma-irradiation models (CBA and C57BL/6 mice) and exhibited a radiomitigating effect in the midlethal range of radiation doses. BP-C2 improved animal survival, protected intestinal crypts and Lgr5+ stem cells and enhanced extramedullar hematopoiesis in the spleen. Neither BP-C2 nor BP-C3 administered to intact animals has effects on blood count or biochemistry, body weight or animal behavior. For pharmacokinetics, the labeled [3H]-BP-Cx-1 was administered intravenously or by gavage once to BALB/c mice. In case of i.v. administration the primary organs of accumulation were the lungs, liver, kidneys and spleen $(50-5000 \mu \mathrm{g} / \mathrm{g}$ tissue). BP-Cx-1 has low oral absolute bioavailability, its concentration in animal organs is several orders of magnitude lower than when administered i.v. (2-18 $\mu \mathrm{g} / \mathrm{g}$ tissue). The present study demonstrated the feasibility of using a lignin derived polyphenolic compositions to diminish the toxic effects of chemotherapy and radiation injury. Both studied compositions provide significant protection effect against intestinal injury caused by chemical (BP-C3) or radiation (BP-C2) exposure. The extensive therapeutic potential of lignin derived polyphenolic compositions is yet to be explored.

This work was supported by Russian Science Foundation (grant \#16-15-00142) and by Russian Foundation for Basic Research (grant \# 18-33-20147).

\section{References}

1. Fedoros E.I. et al. // Oncotarget. 2018. 9(26):18578-18593.

2. Anisimov V.N. et al. // Aging. 2016. 8(9):1866-1875.

3. Panchenko A.V. et al. // Exp Ther Med. 2018. 15(3):3124-3132.

4. Bykov V.N. et al. // Int J Radiat Biol. 2018. 94(2):114-123. 